\title{
ARTICLE OPEN Evidence of spontaneous vortex ground state in an iron-based ferromagnetic superconductor
}

\author{
Wen-He Jiao ${ }^{1,2}$, Qian Tao ${ }^{1}$, Zhi Ren ${ }^{3}$, Yi Liu ${ }^{1}$ and Guang-Han Cao ${ }^{1,4,5}$
}

Spontaneous vortex phase (SVP) is an exotic quantum matter in which quantized superconducting vortices form in the absence of external magnetic field. Although being predicted theoretically nearly 40 years ago, its rigorous experimental verification still appears to be lacking. Here we present low-field magnetic measurements on single crystals of the iron-based ferromagnetic superconductor $\mathrm{Eu}\left(\mathrm{Fe}_{0.91} \mathrm{Rh}_{0.09}\right)_{2} \mathrm{As}_{2}$ which undergoes a superconducting transition at $T_{\mathrm{sc}}=19.6 \mathrm{~K}$ followed by a magnetic transition at $T_{\mathrm{m}}=16.8 \mathrm{~K}$. We observe a characteristic first-order transition from a Meissner state within $T_{\mathrm{m}}<T<T_{\mathrm{sc}}$ to an SVP below $T_{\mathrm{m}}$, under a magnetic field approaching zero. Additional isothermal magnetization and ac magnetic susceptibility measurements at $T \ll T_{\mathrm{sc}}$ confirm that the system is intrinsically in a spontaneous-vortex ground state. The unambiguous demonstration of SVP in the title material lays a solid foundation for future imaging and spectroscopic studies on this intriguing quantum matter.

npj Quantum Materials (2017)2:50; doi:10.1038/s41535-017-0057-0

\section{INTRODUCTION}

Spontaneous vortex (SV) phase, originally predicted by theoretical investigations, ${ }^{1-4}$ is an exotic quantum matter in which superconducting vortices form in the absence of external magnetic field, which can be qualitatively different from those induced by an external field. ${ }^{5}$ While SV state was also predicted to be present in the pseudogap phase of cuprates due to local spins of the paramagnetic phase, ${ }^{6}$ self-induced vortices are mostly generated by an internal magnetic field, $H_{\text {int }}=4 \pi M$, due to the spontaneous magnetization $M$. This means that the prerequisite of realization of an SV state is that superconductivity (SC) coexists with magnetic order, the latter of which at least gives rise to a ferromagnetic component. Such a coexistence is rare because of the antagonism between SC and ferromagnetism (FM). Additional requirements for observation of the SV phenomenon include yet, are not limited to, (1) the SC alone (a hypothetical nonmagnetic analog without any internal field) belongs to the second type with intrinsic lower and upper critical fields $\left(H_{\mathrm{c} 1}^{*}\right.$ and $\left.H_{\mathrm{c} 2}^{*}\right)$; and (2) the internal magnetic field strength lies in the range of $H_{\mathrm{c} 1}^{*}<H_{\mathrm{int}}<H_{\mathrm{c} 2}^{*}$.

Materials that bear both SC and FM generally have distinct superconducting critical temperature $T_{\mathrm{sc}}$ and magnetic transition temperature $T_{\mathrm{m}}$. If $T_{\mathrm{sc}}>T_{\mathrm{m}}$, they are traditionally called "ferromagnetic superconductors" (FSCs). ${ }^{7}$ Otherwise, the terminology "superconducting ferromagnets" (SFMs) are often employed. ${ }^{8}$ According to this classification and, with the consideration of the relative strength between $H_{\mathrm{c} 1}^{*}$ and $H_{\mathrm{int}}$, possible existence of an SV phase is schematically depicted in Fig. 1 for an extremely type-II superconductor in which $H_{\mathrm{c} 2}^{*}(0) \gg H_{\mathrm{c} 1}^{*}(0)$ holds. In the cases of $H_{\text {int }}(0)>H_{\mathrm{c} 1}^{*}(0)$, as shown in the panels (a) and (b), the SV phase can be realized as a ground state. If $H_{\text {int }}(0)<H_{\mathrm{c} 1}^{*}(0)$, however, possible SV phase appears only at finite temperatures above zero, as is seen in the panels (c) and (d).
There have been a few systems that may host an SV state. In the SFMs, Ru-containing cuprates, ${ }^{8,9}$ and U-based UCoGe, ${ }^{10,11}$ which can be categorized into scenarios (c) and (a) in Fig. 1, respectively, were argued to have an SV state on the basis of magnetic measurements. For FSCs, however, evidence of SV phase from bulk magnetic measurements is still lacking, although theoreti$\mathrm{cal}^{12}$ and experimental ${ }^{13}$ investigations suggested an SV state in the weakly ferromagnetic superconductor $\mathrm{ErNi}_{2} \mathrm{~B}_{2} \mathrm{C}$. In fact, rigorous demonstration of a bulk SV phase by magnetic measurements is challenging primarily because an external field, which by itself induces vortices and, possibly changes the magnetic state, has to be applied. In general, one needs to demonstrate the existence of SV state as the external field approaches zero, which requires a sufficiently high measurement precision. This issue becomes more stringent in the cases above where the internal field generated by the small ferromagnetic component is very weak (e.g., the spontaneous magnetization of UCoGe is $\sim 0.04 \mu_{\mathrm{B}} / \mathrm{U}$, corresponding to $\sim 30$ Oe field). Furthermore, the magnetic measurements always encounter the interferences of ferromagnetic domains. ${ }^{11}$

As was first pointed out by $\mathrm{Ng}$ and Varma, ${ }^{12}$ nevertheless, the SV phase can be manifested by the unique first-order phase transition from a Meissner state to an SV phase, which can be possibly seen in cases of Fig. 1b-d. The first-order transition is expected to accompany with a thermal hysteresis that may be easily captured experimentally. Indeed, a thermal hysteresis in magnetic susceptibility was observed in the $\mathrm{SFM} \mathrm{RuSr}_{2} \mathrm{GdCu}_{2} \mathrm{O}_{8}$, which is interpreted as a characteristic of SV state. ${ }^{9}$ However, the observed phenomenon was sample dependent and, the polycrystalline samples employed expose the flaw: the magnetic-flux pinning by grain boundaries might also account for the phenomenon. ${ }^{14}$ Therefore, detection of a Meissner-to-SV transition should be done at least using single crystalline samples.

\footnotetext{
${ }^{1}$ Department of Physics, Zhejiang University, Hangzhou 310027, P. R. China; ${ }^{2}$ Department of Physics, Zhejiang University of Science and Technology, Hangzhou 310023, P. R.

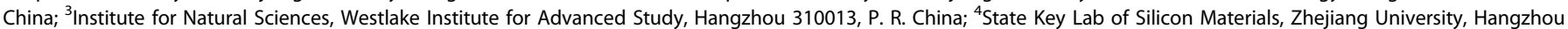
310027, P. R. China and ${ }^{5}$ Collaborative Innovation Centre of Advanced Microstructures, Nanjing 210093, P. R. China Correspondence: Guang-Han Cao (ghcao@zju.edu.cn)
}

Received: 26 May 2017 Revised: 26 July 2017 Accepted: 9 August 2017

Published online: 04 September 2017 


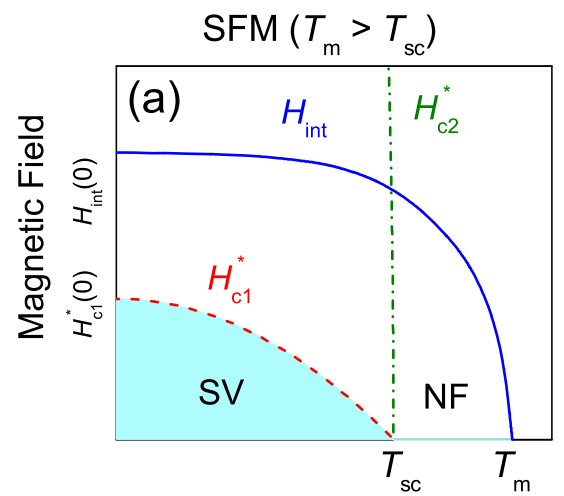

Temperature

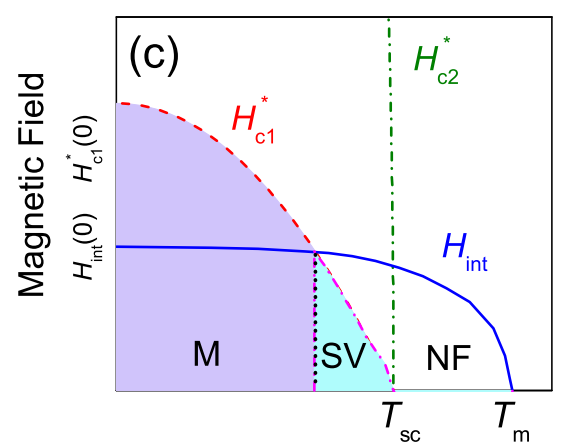

Temperature

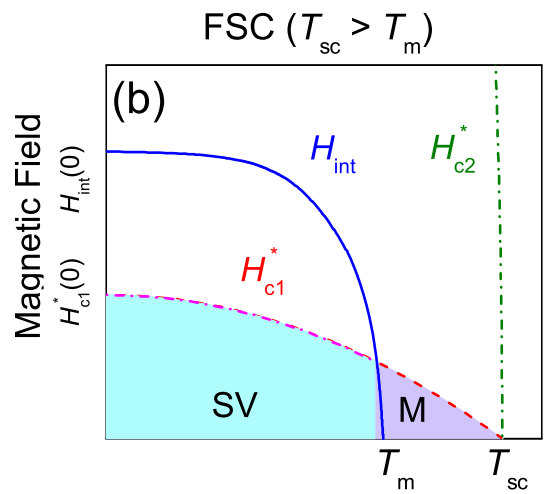

Temperature

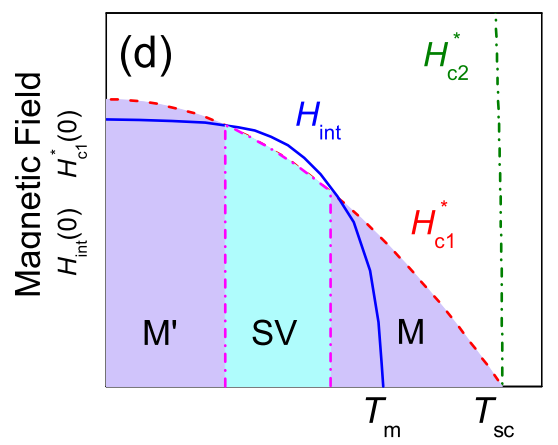

Temperature

Fig. 1 Classification of magnetic superconductors that may host the spontaneous vortex (SV) phase. The left panels show superconducting ferromagnets (SFMs), while the right panels describe ferromagnetic superconductors (FSCs). $\mathbf{a}, \mathbf{b}, H_{\text {int }}(0)>H_{\mathrm{c} 1}^{*}(0) ; \mathbf{c}, \mathbf{d}, H_{\mathrm{c} 1}^{*}(0)>H_{\mathrm{int}}(0)$. See the text for the definitions of $H_{\mathrm{int}}$ and $H_{\mathrm{c} 1}^{*}$. M and $\mathrm{M}^{\prime}$ denote Meissner states, and NF stands for a non-superconducting ferromagnetic state. Here the upper critical fields, $H_{\mathrm{c} 2}^{*}(0)$, are assumed to be much larger than $H_{\mathrm{c} 1}^{*}(0)$ and $H_{\mathrm{int}}(0)$

In this context, the recently discovered FSCs in doped $\mathrm{EuFe}_{2} \mathrm{As}_{2}$ systems ${ }^{15}$ which show a remarkable coexistence of SC and strong FM in a broad temperature range (note that the temperature window for probing an SV phase is mostly below $2.5 \mathrm{~K}$ in previous systems ${ }^{10,11,13}$, provide a desirable platform to look into the SV state. Through either $\mathrm{P}$ doping at As site ${ }^{16}$ or transition-metal (such as Ru, Co, Rh, and Ir) doping at Fe site, ${ }^{17-21} \mathrm{SC}$ can be induced with a $T_{\text {sc }}$ between 20 and $30 \mathrm{~K}$, and the $\mathrm{Eu}^{2+}$ spins (with $S=7 / 2$ ) become ferromagnetically ordered at $T_{\mathrm{m}}$ a few kelvins lower. Although there were debates on details of the magnetic order, $^{15,}{ }^{22-26}$ recent $x$-ray resonant magnetic scattering and neutron diffraction studies ${ }^{27-30}$ show that the $\mathrm{Eu}^{2+}$ spins always align ferromagnetically along the $c$ axis with an ordered moment of about $7 \mu_{\mathrm{B}}$. The ferromagnetic ordering gives rise to a large spontaneous magnetization that generates an internal field of $H_{\text {int }} \approx 9000$ Oe along the $c$ axis, well above the expected $H_{\mathrm{c} 1}^{*}(0)$ of $\sim 150$ Oe. $^{31}$ Additional important advantage of the iron-based FSC is that the high-quality single crystals are easily accessible. ${ }^{17-20}$ Note that the internal-field direction induces superconducting vortices within the FeAs layers. As such, the magnetic measurements can be limited to those under external fields parallel to the $c$ axis, which greatly simplifies the interpretation of the measurement result.

\section{RESULTS}

We employed an optimally Rh-doped single crystal of Eu $\left(\mathrm{Fe}_{0.91} \mathrm{Rh}_{0.09}\right)_{2} \mathrm{As}_{2}$ with $T_{\mathrm{sc}}=19.6 \mathrm{~K}, T_{\mathrm{m}}=16.8 \mathrm{~K}$, and a saturation magnetization $M_{\text {sat }}=6.5 \mu_{\mathrm{B}} / \mathrm{Eu} .{ }^{19}$ The saturation magnetization is close to $g S=7.0$, which tells that the $\mathrm{Eu}^{2+}$ spins align ferromagnetically, similar to other Eu-containing $\mathrm{FSC}^{27-30}$ as shown in Fig. 2a. The superconducting transition of in-plane resistivity is plotted in Fig. 2b. The relatively broadened resistive transition seems to be related to the Eu-spin exchange field which suppresses the $T_{\mathrm{sc}}$ value (note that $T_{\mathrm{sc}}$ is $21.9 \mathrm{~K}$ for the optimally $\mathrm{Rh}$-doped $\mathrm{SrFe}_{2} \mathrm{As}_{2}{ }^{32}$ ). Below $T_{\mathrm{m}}$, the ferromagnetic ordering leads to a re-appearance of resistivity [Fig. 2b]. The maximum of the reentrant resistivity is only $1 / 40$ the normal-state value, indicating that it is by no means a recovery of the normal state, instead, it is associated with the SV formation. Specifically speaking, the revival of resistivity comes from the vortex flow in an SV liquid state. With decreasing temperature, $H_{\mathrm{irr}}^{*}$ surpasses $H_{\mathrm{int}}$ as shown in Fig. $2 \mathrm{c}$, making the vortices frozen, hence zero-resistance state is achieved below $\sim 8 \mathrm{~K}$. Note that the SV scenario naturally explains various resistivity states below $T_{\mathrm{m}}{ }^{15-21}$ some of which show absence of the resistivity reentrance, ${ }^{20,} 23-25$ depending on the doping levels and physical pressures. As is seen, the absence of reentrant behaviour is more easily to be observed in P-doped $\mathrm{EuFe}_{2} \mathrm{As}_{2}{ }^{23-25}$ where $T_{\mathrm{sc}}$ is significantly higher than $T_{\mathrm{m}}$ such that $H_{\mathrm{irr}}^{*}>H_{\text {int }}$ is satisfied.

The dc magnetic susceptibility shows a kink for the field-cooling (FC) protocol and a peak for the zero-field-cooling (ZFC) protocol at $T_{\mathrm{m}}$, as shown in Fig. $2 \mathrm{~d}$. This can be interpreted as the formation of antiparallel ferromagnetic domains. ${ }^{20}$ Because of the proximity between SC and FM, the superconducting transition is not distinctly seen in the dc magnetic measurements (although it was directly observable at very low fields ${ }^{20}$ ). Nevertheless, $X_{c}^{\mathrm{FC}}$ and $X_{c}^{\mathrm{ZFC}}$ bifurcate just at $T_{\mathrm{sC}}$ owing to the magnetic-flux pinning effect. The superconducting magnetic shielding effect below $T_{\mathrm{sc}}$ is confirmed by the following ac susceptibility measurement.

Since the internal field generated by the $\mathrm{Eu}^{2+}$-spin FM is much stronger than the expected $H_{\mathrm{c} 1}^{*}(0)$, as described above, the SV state is stabilized once the FM develops. On the other hand, the internal field vanishes for $T>T_{\mathrm{m}}$, hence it is in a Meissner state at 

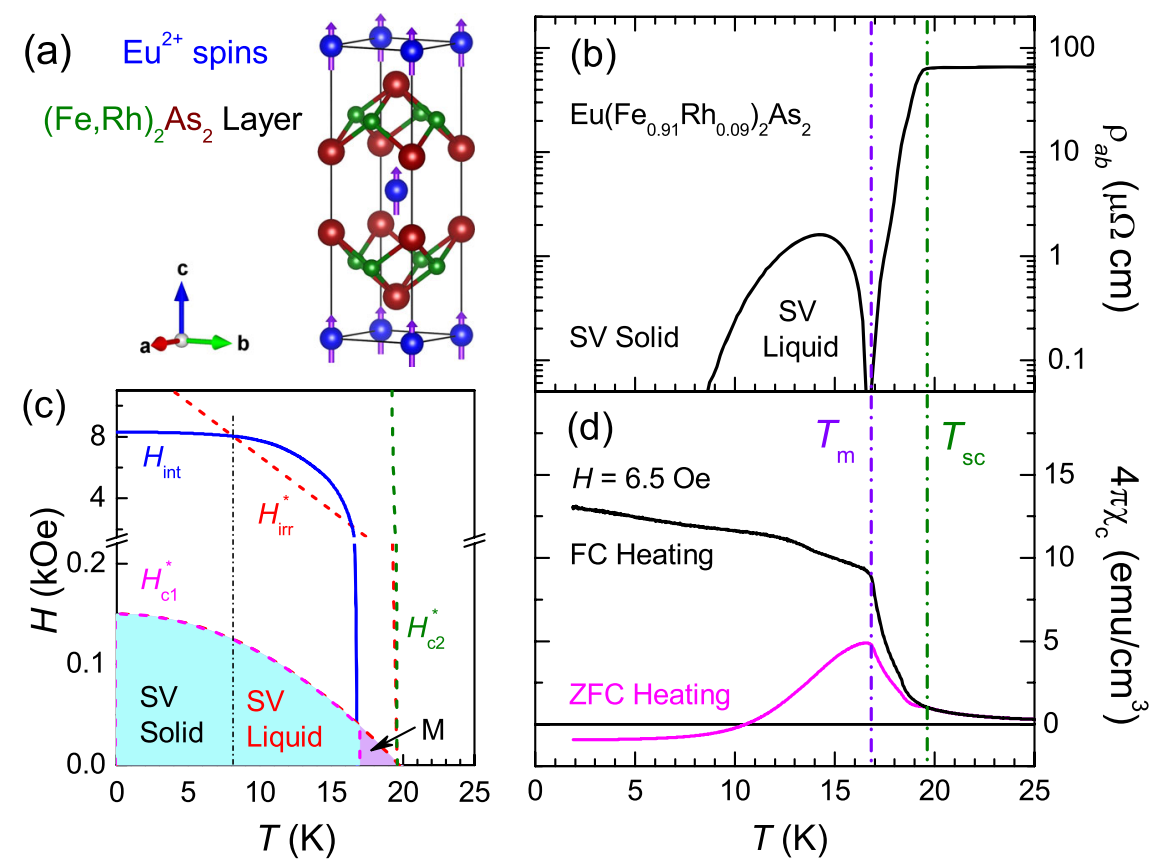

Fig. 2 Characteristic of the ferromagnetic superconductor $\mathrm{Eu}\left(\mathrm{Fe}_{0.91} \mathrm{Rh}_{0.09}\right)_{2} \mathrm{As}_{2}$ in relation with a spontaneous vortex phase. a The crystal and magnetic structure. $\mathbf{b}$ The superconducting resistive transition at $T_{\mathrm{sc}}$, followed by a resistivity revival below $T_{\mathrm{m}}$ (note the logarithmic scale for resistivity). c Schematic $H-T$ diagram showing the internal field $H_{\text {int }}$ (solid blue line), in comparison with the hypothetical irreversible field $H_{\text {irr }}^{*}$ (assuming $H_{\mathrm{int}}=0$ ) as well as the hypothetical lower and upper critical fields, $H_{\mathrm{c} 1}^{*}$ and $H_{\mathrm{c} 2}^{*}$. SV and M denote the spontaneous-vortex phase and the Meissner state, respectively. $\mathbf{d}$ Temperature dependence of the dc magnetic susceptibility measured while heating up, with both fieldcooled (FC) and zero-field-cooled (ZFC) histories. The demagnetization effect has been taken into account

zero external field in the temperature range $T_{\mathrm{m}}<T<T_{\mathrm{sc}}$, as shown in Fig. 2c. Therefore, a transition from the Meissner state to the SV phase definitely occurs as temperature decreases. During the transition, the spontaneous vortices (SVs) suddenly penetrate the crystal's interior, which gives rise to a unique first-order transition with a magnetization discontinuity at around $T_{m}$ for the ideal case with single magnetic domain. In the case of a large sample with multi-domains, nevertheless, a "continuous" change with an obvious thermal hysteresis is expected because of the latent heat in the first-order transition. The possible thermal hysteresis from the domain-wall depinning can be avoided by employing magnetic fields that are much lower than the coercive field $\left(\sim 200 \mathrm{Oe}^{20}\right)$.

As shown in Fig. 3a, the FC magnetization data indeed show a thermal hysteresis in the vicinity of $T_{m}$, demonstrating the nature of first-order transition. In the cooling process, Meissner state is first stabilized, which expectedly gives a lower value of magnetization because of Meissner effect (For the SFM RuSr ${ }_{2} \mathrm{Gd}$ $\mathrm{Cu}_{2} \mathrm{O}_{8}$ where the SV state is stabilized at the high-temperature side, in contrast, the FCC curve has a larger magnetic susceptibility. ${ }^{9}$ ). On the other hand, In the $\mathrm{FCH}$ process from $T_{\mathrm{m}}$ to $T_{\mathrm{sC}}$ some "superheated" spontaneous vortices survive accompanying with the "polarization" of Eu spins until $T_{\mathrm{sc}}$, which gives rise to a higher magnetization value. The hysteresis regime extends up to $T_{\text {sc }}$ suggesting that the SV state could be stabilized by the Eu-spin ferromagnetic fluctuations. The magnetization differences of the cooling and warming data, $\Delta M_{C}=M_{\mathrm{FCC}}-M_{\mathrm{FCH}}$, are plotted in Fig. 3b. One sees that $\Delta M_{c}$ drops at $T_{\mathrm{sc}}$ and it increases rapidly till $T_{\mathrm{m}}$. The maximum of $\left|\Delta M_{c}\right|$ increases with the applied field. Figure $3 c$ plots the $\Delta M_{c}$ value at $T_{m}\left(\Delta M_{c}^{T_{m}}\right)$ as a function of external field. Remarkably, $\Delta M_{c}^{T_{m}}$ is exactly proportional to the field (note that the field accuracy is self-checked by the fielddependent magnetization at $30 \mathrm{~K}$ shown on the right axis). In fact, $\Delta M_{c}$ can be fully scaled with the applied field, as shown in Fig. $3 d$. Here we emphasize that the thermal hysteresis is always observable, even at very low magnetic fields, for different pieces of the sample. By contrast, no thermal hysteresis is seen in overdoped samples where only a ferromagnetic transition takes place. This further rules out the possibility that the domain-wall depinning could be responsible for the large thermal hysteresis.

The magnetization difference at $T_{\mathrm{m}}, \Delta M_{c}^{T_{m}}$, can be understood as follows. For $T \rightarrow T_{\mathrm{m}}^{-}$(FCH data), the SV state dominates. The magnetic contribution of SVs is always accompanied with the ferromagnetic domains. Owing to the existence of multi-domain, the magnetic fluxes from SVs cancels out at zero field, ${ }^{11}$ and with applying fields, the moment appears to be proportional to the external field. When temperature exceeds $T_{\mathrm{m}}$, SVs still survive (superheating effect) although the FM vanishes. Namely, the external magnetic field penetrates the sample where superconducting layers contain SVs and, the $\mathrm{Eu}^{2+}$ spins are basically in the Curie-Weiss paramagnetic state [see the two right-side cartoons in Fig. 3a]. Thus, the FCH magnetic susceptibility at $T_{\mathrm{m}}$ is approximately equal to the Curie-Weiss paramagnetic susceptibility, i.e., $X_{\mathrm{FCH}}^{T_{\mathrm{m}}} \approx X_{\mathrm{CW}}^{T_{\mathrm{m}}}$. For $T \rightarrow T_{\mathrm{m}}^{+}$(FCC data), on the other hand, the Meissner state dominates, which gives an additional diamagnetic susceptibility of $X_{s c}^{T_{m}}$, yielding $X_{F C C}^{T_{m}} \approx X_{c W}^{T_{m}}+X_{s c}^{T_{m}}$. Thus we have, $\Delta X_{c}^{T_{m}}=X_{F C C}^{T_{m}}-X_{F C H}^{T_{m}} \approx X_{s c}^{T_{m}}$, which simply reflects the superconducting magnetic expulsion (see the cartoon pictures). The Meissner volume fraction can be estimated to be, $4 \pi \Delta M_{c}^{T_{m}} / H \approx 15 \%$, which is not surprising because of the unavoidable flux pinning effect.

Above we demonstrate the first-order transition from a Meissner state to an SV phase with decreasing temperature. This suggests that the SV phase represents the ground state in Eu $\left(\mathrm{Fe}_{0.91} \mathrm{Rh}_{0.09}\right)_{2} \mathrm{As}_{2}$. If this is the case, one expects that the lower critical field at zero temperature, $H_{\mathrm{c} 1}(0)$, would be zero. ${ }^{10-12}$ Figure 4 shows the low-temperature isothermal magnetization, $M_{c}(H)$, for $\mathrm{Eu}\left(\mathrm{Fe}_{0.91} \mathrm{Rh}_{0.09}\right)_{2} \mathrm{As}_{2}$, in comparison with that of the nonmagnetic superconducting analog, $\mathrm{Ba}\left(\mathrm{Fe}_{0.9} \mathrm{Co}_{0.1}\right)_{2} \mathrm{As}_{2}$. The latter shows an essentially linear $M_{c}(H)$ since the applied fields are much lower than the $H_{\mathrm{c} 1}(0)$. In contrast, $\mathrm{Eu}\left(\mathrm{Fe}_{0.91} \mathrm{Rh}_{0.09}\right)_{2} \mathrm{As}_{2}$ displays a non-linear virgin $M_{c}(H)$ curve and an obvious magnetic 

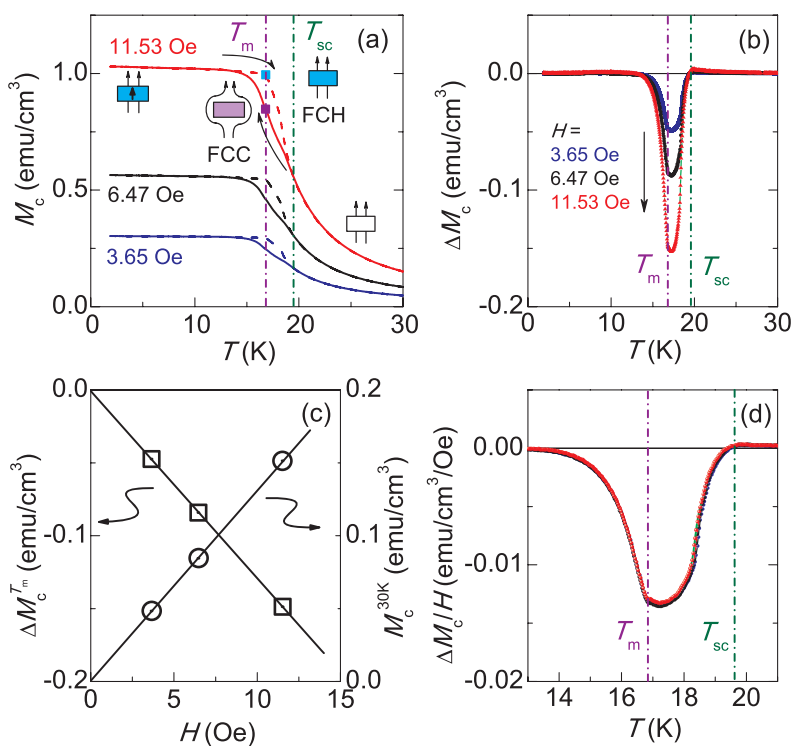

Fig. 3 Evidence of the first-order transition from a Meissner state to a spontaneous vortex phase with decreasing temperature in $\mathrm{Eu}$ $\left(\mathrm{Fe}_{0.91} \mathrm{Rh}_{0.09}\right)_{2} \mathrm{As}_{2}$ crystals. a Field-cooling magnetization $\left(M_{\mathrm{c}}\right)$ on both heating $(\mathrm{FCH})$ and cooling $(\mathrm{FCC})$ processes under magnetic fields along the $c$ axis. The rectangles with arrows schematically represent different statuses of sample in the presence of external field (multidomains and pinned fluxes are not shown). See the text for the inserted cartoon pictures $\mathbf{b}$ The magnetization difference $\Delta M_{c}$ between the FCH and FCC data. c $\Delta M_{c}$ at $T_{m}$ (left axis) and $M_{c}$ at $30 \mathrm{~K}$ (right axis) as functions of the applied field. d $\Delta M_{C} / H$ vs. $T$ in an expanded temperature range

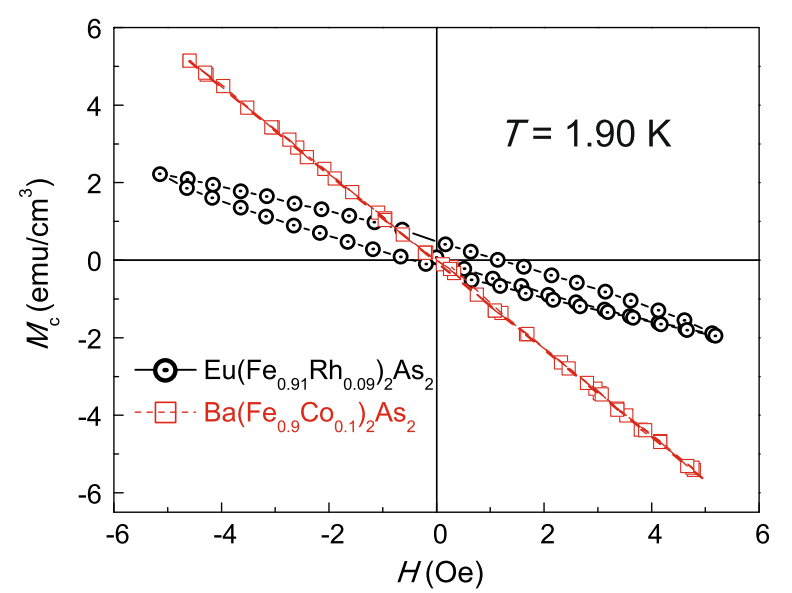

Fig. 4 Isothermal magnetization curves at $1.90 \mathrm{~K}$ under magnetic fields parallel to the $c$ axis for $\mathrm{Eu}\left(\mathrm{Fe}_{0.91} \mathrm{Rh}_{0.09}\right)_{2} \mathrm{As}_{2}$ and $\mathrm{Ba}$ $\left(\mathrm{Fe}_{0.9} \mathrm{Co}_{0.1}\right)_{2} \mathrm{As}_{2}$

hysteresis loop. This means that, in addition to the superconducting magnetic shielding effect, the external field always penetrates the sample, even if the field is around zero. In other words, $\mathrm{Eu}\left(\mathrm{Fe}_{0.91} \mathrm{Rh}_{0.09}\right)_{2} \mathrm{As}_{2}$ is intrinsically in a mixed state below $T_{\mathrm{m}}$.

Another piece of evidence for the mixed state at zero field comes from the ac magnetic susceptibility measurements. As shown in the main panel of Fig. 5 , one can clearly distinguish $T_{\mathrm{sc}}$ and $T_{\mathrm{m}}$ from the real part of the ac susceptibility, $X^{\prime}$. The magnetic shielding effect below $T_{\mathrm{sc}}$ is much more obvious than that of the dc magnetic measurement above. The imaginary part of the susceptibility, $x^{\prime \prime}$, which is sensitive to dissipations, shows two sharp peaks below $T_{\mathrm{sc}}$ and $T_{\mathrm{m}}$, respectively. An additional large

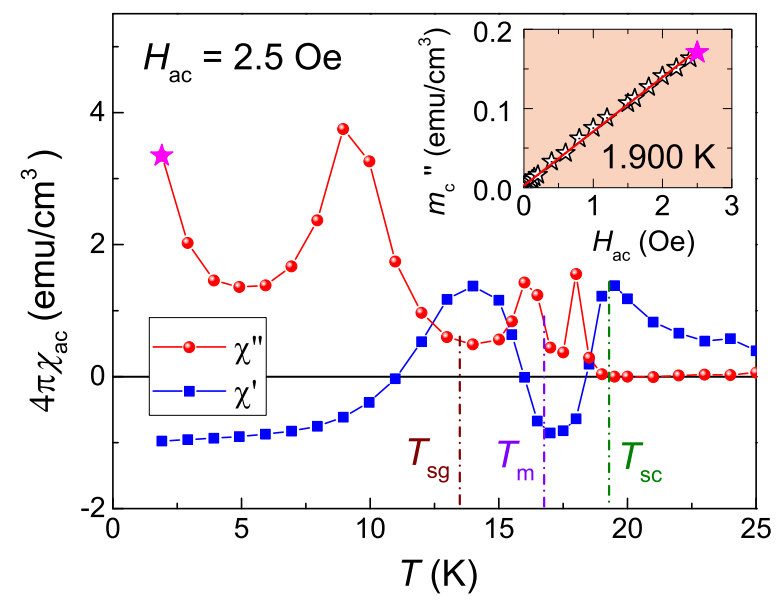

Fig. 5 Temperature dependence of real and imaginary parts $\left(x^{\prime}\right.$ and $\left.x^{\prime \prime}\right)$ of ac susceptibility at zero dc magnetic field. The amplitude of the driving ac magnetic field (along the $c$ axis) is, $H_{a c}=2.5$ Oe. The demagnetization effect has been taken into consideration. $T_{\mathrm{sc}}, T_{\mathrm{m}}$, and $T_{s g}$ are the superconducting, ferromagnetic, and spin-glass transition temperatures, respectively. The inset plots the imaginary part of the ac magnetization at $1.9 \mathrm{~K}$ as a function of $H_{\mathrm{ac}}$. The solid line is the linear fit

broad peak appears below the re-entrant spin-glass temperature $T_{\text {sg }} \approx 13.5 \mathrm{~K}^{19}$ Note that this $\chi^{\prime \prime}$ peak may also be contributed from the SV liquid-to-solid transition.

Remarkably, the $\chi^{\prime \prime}$ value at the lowest temperature of $1.90 \mathrm{~K}$ in our measurements remains considerably high at the driving field $H_{\mathrm{ac}}=2.5 \mathrm{Oe}$, verifying that it is in a mixed state. To examine if there is a lower limit of the ac field, we performed a fielddependent ac magnetization measurement, the imaginary part $\left(m_{c}^{\prime \prime}\right)$ of which is shown in the inset of Fig. 5 . One sees that $m_{c}^{\prime \prime}$ is exactly proportional to $H_{a c}$. According to the critical-state model, ${ }^{33}$ $m_{c}^{\prime \prime}$ will be zero for $H_{\mathrm{ac}}<H_{\mathrm{c} 1}$; while $m_{c}^{\prime \prime}=\beta\left(H_{\mathrm{ac}}-H_{\mathrm{c} 1}\right)^{2} / H_{\mathrm{ac}}(\beta$ is the sample's geometrical factor) for $H_{\mathrm{ac}}>H_{\mathrm{c} 1}$. Both the non-zero $m_{c}^{\prime \prime}$ and the linearity of $m_{c}^{\prime \prime}\left(H_{\mathrm{ac}}\right)$ through the origin indicate that $H_{\mathrm{c} 1}$ must be zero. Similar observation is seen in the SFM UCoGe. ${ }^{11}$

\section{DISCUSSION}

The above results allow us to arrive at the following picture for the $\mathrm{Eu}\left(\mathrm{Fe}_{0.91} \mathrm{Rh}_{0.09}\right)_{2} \mathrm{As}_{2} \mathrm{FSC}$ in the absence of external magnetic field. At $T>T_{\mathrm{sc}}$, the $\left[(\mathrm{Fe}, \mathrm{Rh})_{2} \mathrm{As}_{2}\right]^{2-}$ and $\mathrm{Eu}^{2+}$ layers are Pauli paramagnetic and Curie-Weiss paramagnetic, respectively. When cooled below $T_{\mathrm{sc}}$, the $\left[(\mathrm{Fe}, \mathrm{Rh})_{2} \mathrm{As}_{2}\right]^{2-}$ layers become superconducting, showing a Meissner state coexisting with the Curie-Weiss paramagnetism of $\mathrm{Eu}^{2+}$ spins. With further decreasing temperature to below $T_{\mathrm{m}}$, the $\mathrm{Eu}^{2+}$ spins are ferromagnetic ordered along the $c$ axis, which generates an internal field far above the expected $H_{\mathrm{c} 1}^{*}(0)$. Superconducting vortices then form spontaneously in the $\left[(\mathrm{Fe}, \mathrm{Rh})_{2} \mathrm{As}_{2}\right]^{2-}$ layers. In the temperature range of $8 \mathrm{~K}<T<T_{\mathrm{m}}$, the SVs are mobile, which leads to the revival of resistance. The subsequent solidification of the SVs below $8 \mathrm{~K}$ gives rise to the zero-resistance state. Therefore, the ground state is an SV solid, which reconciles $\mathrm{SC}$ and $\mathrm{FM}$ in $\mathrm{Eu}\left(\mathrm{Fe}_{0.91} \mathrm{Rh}_{0.09}\right)_{2} \mathrm{As}_{2}$.

Finally we note that, apart from the formation of the SV phase, an alternative that allows to reconcile the SC and FM is the socalled Fulde-Ferrell-Larkin-Ovchinnikov (FFLO) state characterized by a spatial modulation of the superconducting order parameter. ${ }^{34,35}$ Nevertheless, in general, realization of an FFLO state at zero external field needs more rigorous conditions. Among them are Pauli-limited $H_{\mathrm{c} 2}^{\perp}$ (for $H \perp a b$ ) with a large Maki parameter and clean limit for the $\mathrm{SC}$, which cannot be satisfied in the present system. The $H_{\mathrm{c} 2}^{\perp}(T)$ curve in $\mathrm{Eu}\left(\mathrm{Fe}_{0.91} \mathrm{Rh}_{0.09}\right)_{2} \mathrm{As}_{2}$ keeps linear down to $0.2 T_{\mathrm{sc},}{ }^{19}$ indicating that the orbital-limiting effect dominates. 
Besides, the large residual resistivity $(\sim 60 \mu \Omega \mathrm{cm})$ as well as the small residual resistivity ratio ( $R R R=2.6)^{19}$ suggests a dirty limit. Both properties actually favor the SV scenario. Nevertheless, here we note that the recently discovered 1144-type $\mathrm{FSC}^{36,37}$ could be the candidate for an FFLO state, because their nonmagnetic analog, $\mathrm{CaKFe}_{4} \mathrm{As}_{4}$, indeed shows a large Maki parameter together with a clean limit for the $\mathrm{SC}^{38}$

In summary, we have studied the low-field magnetic properties for the iron-based ferromagnetic superconductor $\mathrm{Eu}\left(\mathrm{Fe}_{0.91} \mathrm{R}\right.$ $\left.\mathrm{h}_{0.09}\right)_{2} \mathrm{As}_{2}$. We observed a remarkable thermal hysteresis around the ferromagnetic transition in the superconducting state, even under a vanishingly small field, demonstrating the unique firstorder transition from a Meissner state to an SV phase. The SV ground state is further corroborated by the non-linear virgin dc magnetization as well as the non-zero imaginary part of ac magnetic susceptibility under extremely low external fields at $T \ll T_{\mathrm{sc}}$. The unambiguous demonstration of the SV ground state in the iron-based FSC lays a solid foundation for future studies. For example, it is of great interest to see whether the SV solid behaves like a glassy or a lattice state. The imaging observations such as magnetic-force microscopy as well as the small-angle neutron scattering technique may help to clarify this interesting issue.

\section{METHODS}

Crystal growth

High-quality crystals of $\mathrm{Eu}\left(\mathrm{Fe}_{0.91} \mathrm{Rh}_{0.09}\right)_{2} \mathrm{As}$, were grown by a self-flux method. ${ }^{19,}{ }^{20}$ First, mixtures of Eu (99.9\%), Fe (99.998\%), Rh (99.9\%), and As (99.999\%) powders in a molar ratio of Eu:Fe:Rh:As $=1: 4.4: 0.6: 5$ reacted at $973 \mathrm{~K}$ for $24 \mathrm{~h}$ in a sealed evacuated quartz ampoule. The precursor was ground, and then was loaded into an alumina crucible. The crucible was sealed in a stainless steel tube by arc welding under an atmosphere of argon. The assembly was subsequently heated up to $1573 \mathrm{~K}$ and, holding for $5 \mathrm{~h}$, in a muffle furnace with the flow of argon gas. The crystal growth took place during the slow cooling down to $1223 \mathrm{~K}$ at the rate of $4 \mathrm{~K} / \mathrm{h}$. Large crystals with typical size of $3 \times 3 \times 0.5 \mathrm{~mm}^{3}$ were harvested.

\section{Structural and compositional characterizations}

We checked the as-grown crystal flakes by $x$-ray diffraction using a PANAlytical $x$-ray diffractometer (using $\mathrm{Cu} K_{\alpha 1}$ monochromatic radiation) at room temperature. All the crystals show only (00/) reflections with even I values, similar to the previous report. ${ }^{19}$ The $c$ axis is then determined to be $12.016(1) \AA$. The crystal structure is analogous to $\mathrm{EuFe}_{2} \mathrm{As}_{2}(c=12.136 \AA),{ }^{39}$ yet it consists of superconducting $\left[(\mathrm{Fe}, \mathrm{Rh})_{2} \mathrm{As}_{2}\right]^{2-}$ layers separated by magnetic $\mathrm{Eu}^{2+}$ ions. The full width at half maximum (FWHM) of the reflection peaks is typically $2 \theta=0.06^{\circ}$, verifying the high quality of the crystals. The real composition of the crystal was determined by energy dispersive $\mathrm{x}$-ray spectroscopy, which gives the chemical formula of Eu $\left(\mathrm{Fe}_{0.91} \mathrm{Rh}_{0.09}\right)_{2} \mathrm{As}_{2}$

\section{Physical properties}

The electrical and magnetic properties of the $\mathrm{Eu}\left(\mathrm{Fe}_{0.91} \mathrm{Rh}_{0.09}\right)_{2} \mathrm{As}_{2}$ crystals were reported previously ${ }^{19}$ which demonstrate a superconducting transition at $T_{\mathrm{sc}}=19.6 \mathrm{~K}$, followed by a ferromagnetic transition at $T_{\mathrm{m}}=$ $16.8 \mathrm{~K}$. The isothermal magnetization loops below $T_{\mathrm{m}}$ show characteristic features for both FM and SC. The saturation magnetization achieves $M_{\text {sat }}=$ $6.5 \mu_{\mathrm{B}} / \mathrm{Eu}$, confirming that the Eu spins align ferromagnetically.

\section{Low-field magnetic measurements}

We selected a free-standing crystal for all the measurements in this paper. Magnetic measurements were carried out on a Quantum Design Magnetic Property Measurement System. The residual field in the superconducting magnet, after being removed by a degaussing procedure prior to the measurements, is less than \pm 0.05 Oe. The crystal was carefully mounted into the sample holder with the applied field perpendicular to the crystal plate, such that the external field is either parallel or antiparallel to the internal field. The FC data were collected in both heating and cooling procedures. In the ac susceptibility measurement, the frequency was set to $1.0 \mathrm{~Hz}$. The demagnetization effect is taken into account on the basis of the sample's geometry in respect to the field direction.
Data availability

The data that support the findings of this study are available from the corresponding author (G.H.C.) upon reasonable request.

\section{ACKNOWLEDGEMENTS}

This work is supported by the National Science Foundation of China (Nos.11474252 and 11504329), the National Key Research and Development Program of China (No.2016YFA0300202), and the Fundamental Research Funds for the Central Universities of China.

\section{AUTHOR CONTRIBUTIONS}

W.H.J. and Y.L. grew and characterized the crystals. W.H.J. and Q.T. performed the magnetic measurements. W.H.J., Z.R. and G.H.C. analyzed and interpreted the data, and wrote the paper. This work was coordinated and designed by G.H.C.

\section{ADDITIONAL INFORMATION}

Competing interests: The authors declare no competing financial interests.

Publisher's note: Springer Nature remains neutral with regard to jurisdictional claims in published maps and institutional affiliations.

\section{REFERENCES}

1. Blount, E. I. \& Varma, C. M. Electromagnetic effects near the superconductor-toferromagnet transition. Phys. Rev. Lett. 42, 1079-1082 (1979).

2. Tachiki, M. et al. Self-induced vortices in magnetic superconductors. Solid State Commun 34, 19-23 (1980).

3. Greenside, H. S. et al. Possible coexisting superconducting and magnetic states. Phys. Rev. Lett. 46, 49-53 (1981).

4. Kuper, C. G. et al. Ferromagnetic superconductors: a vortex phase in ternary rareearth compounds. Phys. Rev. Lett. 44, 1545-1548 (1980).

5. Radzihovsky, L. et al. "soft" anharmonic vortex glass in ferromagnetic superconductors. Phys. Rev. Lett. 87, 027001 (2001).

6. Weng, Z. Y. \& Muthukumar, V. N. Spontaneous vortex phase in the bosonic resonating valence bond theory. Phys. Rev. B 66, 094509 (2002).

7. Bulaevskii, L. et al. Coexistence of superconductivity and magnetism theoretical predictions and experimental results. Adv. Phys 34, 175-261 (1985).

8. Sonin, E. B. \& Felner, I. Spontaneous vortex phase in a superconducting weak ferromagnet. Phys. Rev. B 57, 14000(R)-14003(R) (1998).

9. Bernhard, $C$. et al. Evidence for a bulk meissner state in the ferromagnetic superconductor $\mathrm{RuSr}_{2} \mathrm{GdCu}_{2} \mathrm{O}_{8}$ from dc magnetization. Phys. Rev. B 61, 14960 (R)-14963(R) (2000)

10. Deguchi, K. et al. Absence of meissner state and robust ferromagnetism in the superconducting state of UCoGe: possible evidence of spontaneous vortex state. J. Phys. Soc. Jpn. 79, 083708 (2010).

11. Paulsen, C. et al. Observation of the meissner-ochsenfeld effect and the absence of the meissner state in UCoGe. Phys. Rev. Lett. 109, 237001 (2012).

12. Ng, T. K. \& Varma, C. M. Spontaneous vortex phase discovered? Phys. Rev. Lett. 78, 330-333 (1997)

13. Chia, E. E. M. et al. Observation of the spontaneous vortex phase in the weakly ferromagnetic superconductor $\mathrm{ErNi}_{2} \mathrm{~B}_{2} \mathrm{C}$ : a penetration depth study. EPL (Europhysics Letters) 73, 772-778 (2006).

14. Papageorgiou, T. P. et al. Magnetization, vortex state and specific heat in the superconducting state of $\mathrm{RuSr}_{2} \mathrm{GdCu}_{2} \mathrm{O}_{8}$. The European Physical Journal B - Condensed Matter and Complex Systems 52, 383-388 (2006).

15. Zapf, S. \& Dressel, M. Europium-based iron pnictides: a unique laboratory for magnetism, superconductivity and structural effects. Reports on Progress in Physics 80, 016501 (2017).

16. Ren, Z. et al. Superconductivity induced by phosphorus doping and its coexistence with ferromagnetism in $\mathrm{EuFe}_{2}\left(\mathrm{As}_{0_{7}} \mathrm{P}_{03}\right)_{2}$. Phys. Rev. Lett. 102, 137002 (2009).

17. Jiang, S. et al. Superconductivity and local-moment magnetism in Eu $\left(\mathrm{Fe}_{0.89} \mathrm{Co}_{0.11}\right)_{2} \mathrm{As}_{2}$. Phys. Rev. B 80, 184514 (2009).

18. Jiao, W.-H. et al. Anisotropic superconductivity in $\mathrm{Eu}\left(\mathrm{Fe}_{0.75} \mathrm{Ru}_{0.25}\right)_{2} \mathrm{As}_{2}$ ferromagnetic superconductor. EPL (Europhysics Letters) 95, 67007 (2011).

19. Jiao, W.-H. et al. Peculiar properties of the ferromagnetic superconductor Eu $\left(\mathrm{Fe}_{0.91} \mathrm{Rh}_{0.09}\right)_{2} \mathrm{As}_{2}$. Supercond. Sci. Technol 30, 025012 (2017).

20. Jiao, W.-H. et al. Anomalous critical fields and the absence of meissner state in $\mathrm{Eu}$ $\left(\mathrm{Fe}_{0.88} \mathrm{Ir}_{0.12}\right)_{2} \mathrm{As}_{2}$ crystals. New. J. Phys. 15, 113002 (2013).

21. Paramanik, U. B. et al. Reentrant superconductivity in $\mathrm{Eu}\left(\mathrm{Fe}_{1-x} \mid \mathrm{r}_{x}\right)_{2} \mathrm{As}_{2}$. J. Phys. Condens. Matt 25, 265701 (2013). 
22. Nowik, I. et al. Coexistence of ferromagnetism and superconductivity: magnetization and mossbauer studies of $\operatorname{EuFe}_{2}\left(\mathrm{As}_{1-x} \mathrm{P}_{x}\right)_{2}$. J. Phys.: Condens. Matt 23, 065701 (2011).

23. Terashima, $\mathrm{T}$. et al. EuFe $\mathrm{As}_{2}$ under high pressure: an antiferromagnetic bulk superconductor. J. Phys. Soc. Jpn. 78, 083701 (2009).

24. Jeevan, H. S. et al. Interplay of antiferromagnetism, ferromagnetism, and superconductivity in $\mathrm{EuFe}_{2}\left(\mathrm{As}_{1-x} \mathrm{P}_{x}\right)_{2}$ single crystals. Phys. Rev. B 83, 054511 (2011).

25. Tokiwa, $Y$. et al. Unique phase diagram with narrow superconducting dome in $\mathrm{EuFe}_{2}\left(\mathrm{As}_{1-x} \mathrm{P}_{x}\right)_{2}$ due to $\mathrm{Eu}^{2+}$ local magnetic moments. Phys. Rev. B 86, 220505 (2012).

26. Zapf, S. et al. EuFe ${ }_{2}\left(\mathrm{As}_{1-x} \mathrm{P}_{x}\right)_{2}$ : Reentrant spin glass and superconductivity. Phys. Rev. Lett. 110, 237002 (2013).

27. Jin, W. T. et al. Magnetic structure of superconducting $\mathrm{Eu}\left(\mathrm{Fe}_{0.82} \mathrm{Co}_{0.18}\right)_{2} \mathrm{As}_{2}$ as revealed by single-crystal neutron diffraction. Phys. Rev. B 88, 214516 (2013).

28. Nandi, S. et al. Coexistence of superconductivity and ferromagnetism in P-doped $\mathrm{EuFe}_{2} \mathrm{As}_{2}$. Phys. Rev. B 89, 014512 (2014).

29. Nandi, S. et al. Magnetic structure of the $\mathrm{Eu}^{2+}$ moments in superconducting $\mathrm{EuFe}_{2}\left(\mathrm{As}_{1-x} \mathrm{P}_{x}\right)_{2}$ with $x=0.19$. Phys. Rev. B 90, 094407 (2014).

30. Jin, W. T. et al. Magnetic ground state of superconducting $E u\left(F e_{0.88} \mid r_{0.12}\right)_{2} A_{2}$ : a combined neutron diffraction and first-principles calculation study. Phys. Rev. $B$ 91, 064506 (2015).

31. Abdel-Hafiez, M. et al. Nodeless superconductivity in the presence of spin-density wave in pnictide superconductors: the case of $\mathrm{BaFe}_{2-x} \mathrm{Ni}_{x} \mathrm{As}_{2}$. Phys. Rev. B 91, 024510 (2015).

32. Han, F. et al. Superconductivity and phase diagrams of the $4 d$ - and $5 d$-metaldoped iron arsenides $\mathrm{SrFe}_{2-x} \mathrm{M}_{x} \mathrm{As}_{2}(M=\mathrm{Rh}, \mathrm{Ir}, \mathrm{Pd}$ ). Phys. Rev. B 80, 024506 (2009).

33. Bean, C. P. Magnetization of high-field superconductors. Rev. Mod. Phys. 36, 31-39 (1964).
34. Fulde, P. \& Ferrell, R. A. Superconductivity in a strong spin-exchange field. Phys. Rev 135, A550-A563 (1964).

35. Larkin, A. I. \& Ovchinnikov, Y. N. Inhomogeneous state of superconductors. Soviet Physics JETP-USSR 20, 762-769 (1965).

36. Liu, Y. et al. Superconductivity and ferromagnetism in hole-doped $\mathrm{RbEuFe}_{4} \mathrm{As}_{4}$. Phys. Rev. B 93, 214503 (2016)

37. Liu, Y. et al. A new ferromagnetic superconductor: $\mathrm{CsEuFe}{ }_{4} \mathrm{As}_{4}$. Sci. Bull 61, 1213-1220 (2016).

38. Meier, W. R. et al. Anisotropic thermodynamic and transport properties of singlecrystalline $\mathrm{CaKFe}_{4} \mathrm{As}_{4}$. Phys. Rev. B 94, 064501 (2016).

39. Ren, Z. et al. Antiferromagnetic transition in $\mathrm{EuFe}_{2} \mathrm{As}_{2}$ : a possible parent compound for superconductors. Phys. Rev. B 78, 052501 (2008).

(c) Open Access This article is licensed under a Creative Commons Attribution 4.0 International License, which permits use, sharing, adaptation, distribution and reproduction in any medium or format, as long as you give appropriate credit to the original author(s) and the source, provide a link to the Creative Commons license, and indicate if changes were made. The images or other third party material in this article are included in the article's Creative Commons license, unless indicated otherwise in a credit line to the material. If material is not included in the article's Creative Commons license and your intended use is not permitted by statutory regulation or exceeds the permitted use, you will need to obtain permission directly from the copyright holder. To view a copy of this license, visit http://creativecommons. org/licenses/by/4.0/.

(c) The Author(s) 2017 